\title{
Intergalactic star formation around NGC 5291
}

\author{
Médéric Boquien ${ }^{1}$, Pierre-Alain Duc ${ }^{1}$, Jonathan Braine ${ }^{2}$, \\ Elias Brinks ${ }^{3}$, Vassilis Charmandaris ${ }^{4}$ and Ute Lisenfeld ${ }^{5}$ \\ ${ }^{1}$ AIM - Unité Mixte de Recherche CEA - CNRS - Université Paris VII - UMR n ${ }^{\circ} 7158$ \\ CEA/Saclay; DSM/DAPNIA/Service d'Astrophysique, CEA/Saclay L'Orme des Merisiers \\ Bat. 709, 91191 Gif-sur-Yvette, France \\ ${ }^{2}$ Observatoire de Bordeaux, UMR 5804, CNRS/INSU, B.P. 89, F-33270 Floirac, France \\ ${ }^{3}$ Center for Astrophysics Research, University of Hertfordshire, College Lane, Hatfield AL10 \\ $9 \mathrm{AB}, \mathrm{UK}$ \\ ${ }^{4}$ Department of Physics, University of Crete, GR-71003, Heraklion, Greece \\ ${ }^{5}$ Dept. de Física Teórica y del Cosmos, Universidad de Granada, Granada, Spain
}

Numerous instances of intergalactic star forming regions have been recently reported (see Duc et al. in this proceedings book). They are fueled by gaseous material expelled from parent galaxies. One spectacular example is the HI ring-like structure around the interacting system NGC 5291 (Malphrus et al. 1997) which hosts numerous HII regions (Duc \& Mirabel 1998). In order to study how star formation proceeds in this specific environment, we have combined ultraviolet (Galex), $\mathrm{H} \alpha, 8 \mu \mathrm{m}$ (Spitzer) and HI (VLA B-array) images of this system.

We have found that, qualitatively, the three star-forming indicators - ultraviolet, $\mathrm{H} \alpha$ and $8 \mu \mathrm{m}$ bands - have a very similar morphology. The normalised infrared emission at $8.0 \mu \mathrm{m}$ which was previously shown to be dominated by PAH bands (Higdon et al. 2006) is comparable to the integrated emission of dwarves of the same metallicity and to the emission of individual HII regions in spirals. The $8.0 \mu \mathrm{m}$ emission in the intergalactic environment is therefore an estimator of the star formation rate (SFR) as reliable as for spirals. There is a clear excess of ultraviolet emission compared to individual HII regions in spirals, i.e. the $[8.0] /[N U V]$ and $[H \alpha] /[N U V]$ flux ratios are on average very low although there are some large variations from one region to the other. While the scatter of $[8.0] /[N U V]$ is largely due to large spatial variations of the dust extinction, as traced by the HI column density, the scatter of $[H \alpha] /[N U V]$ ratio is best explained by age effects. A model of the evolution $[H \alpha] /[N U V]$ with time favours young but already fading quasi-instantaneous starbursts. The total SFR measured in the intergalactic medium surrounding NGC 5291 is up to $1.3 \mathrm{M}_{\odot} \mathrm{yr}^{-1}$, a value typical for spirals, assuming the standard SFR calibrations are valid.

\section{References}

Boquien, M. S. et al., to be submitted to $A \mathscr{E} A$

Duc, P.-A. \& Mirabel, I. 1998, A\&\&A 333, 813

Higdon, S. et al. 2006, ApJ 640, 768

Malphrus, B. et al. 1997, AJ 114, 1427 